\title{
In vivo evaluation of antibacterial activity and wound healing power of a gel based on phenolic compounds extracted from by-products of the wine industry
}

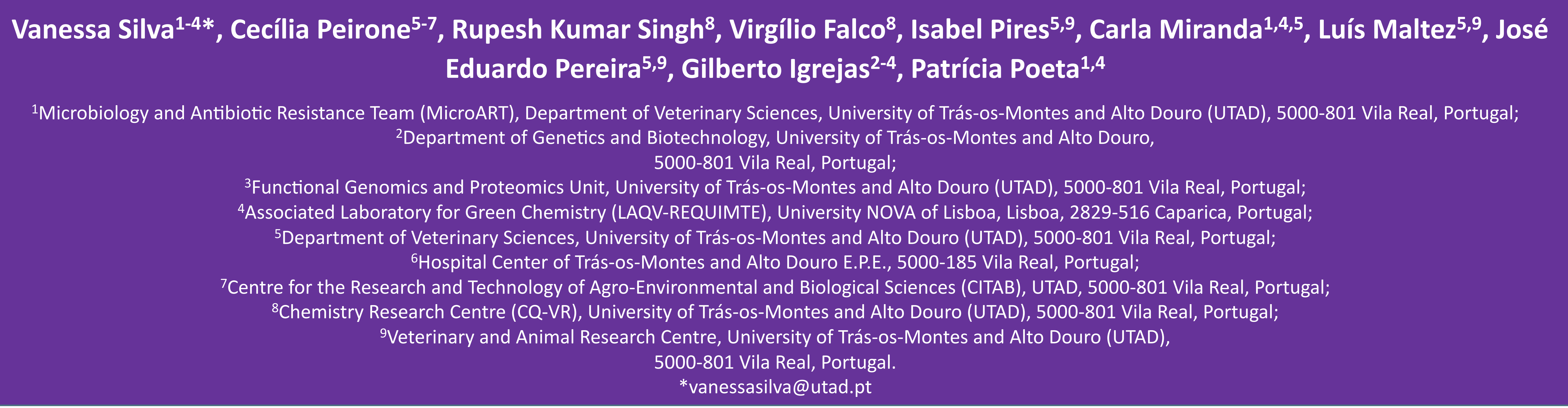

\section{Introduction}

Agro-industrial by-products have been described as potential sources of phenolic compounds, which exhibit several properties, such as, antimicrobial, antioxidant, anti-inflammatory and wound healing. Therefore, we aimed to evaluate the the effect of topical application of a phenolic compounds-base gel on the treatment of skin infected with MRSA and its wound healing capacity in an animal model.

\section{Material and Methods}

The extraction of phenolic compounds from grape's peel, stem and seed was performed using ethanol/water (80:20) mixture. Carbopol gel (0.8\%) was neutralized with triethanolamine and enriched with the extracts. Ten Wistar rats were inoculated by an intradermic injection of MRSA suspension, producing an abscess lesion. After 3 days, the skin epidermis was removed to open the wound (Figure 1). Group 1 (control) received an application of Carbopol gel and Group 2 received an application of Carbopol gel enriched with phenolic extracts (10\%). After the treatment period, skin was collected to quantify colony forming units (CFU) of bacteria and to examine the following histological structures and processes: epidermal regeneration, granulation tissue thickness, fibroblast proliferation, angiogenesis and the presence of inflammatory cells.

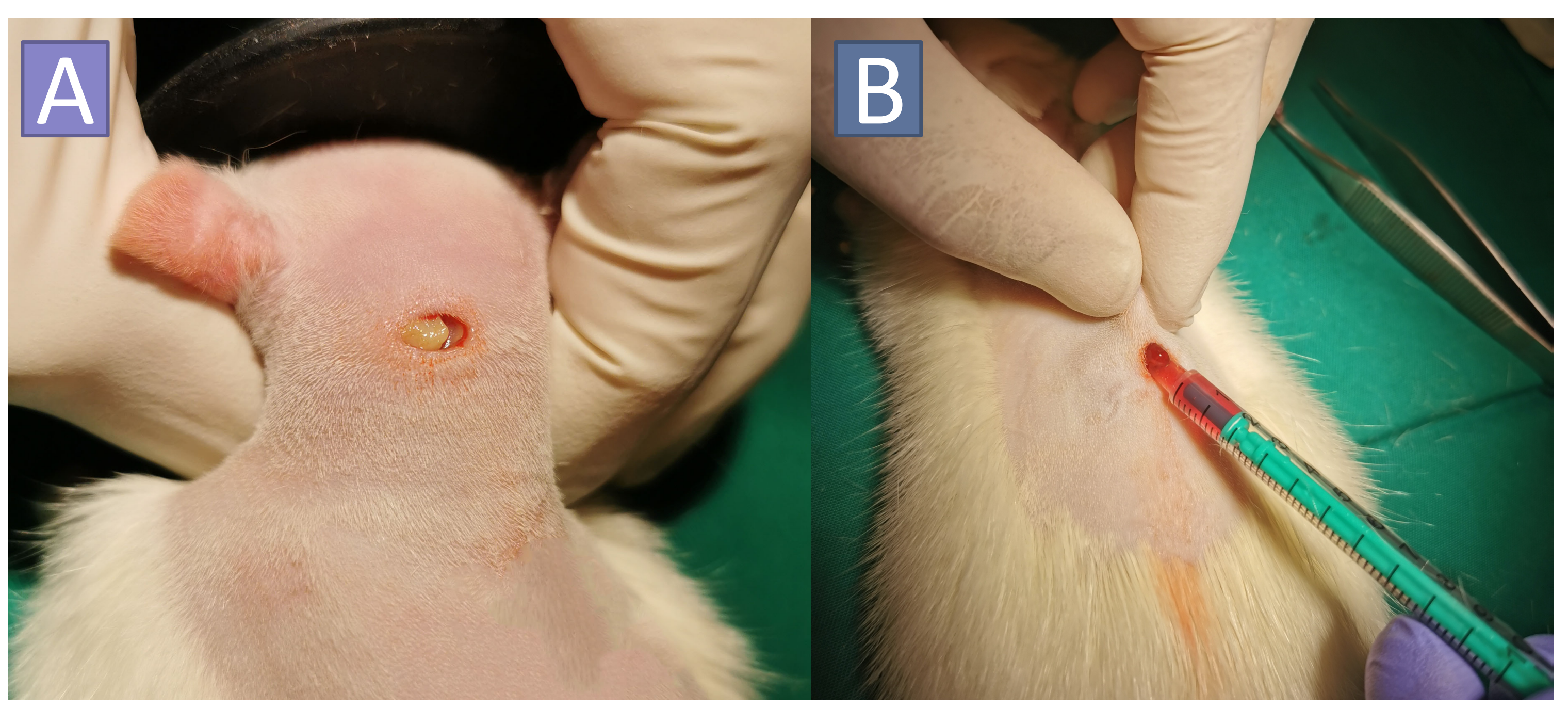

Figure 1. Abscess lesion (A); application of Carbopol gel enriched with phenolic extracts $(B)$.

\section{Results}

Group 1 and 2 presented a similar number of $\mathrm{CFU} / \mathrm{mL}$ (difference not statistically significant) (Figure 2) which is not in line with that obtained from previous in vitro studies in which phenolic extracts present antibacterial activity. Regarding the histological evaluation, the presence of histological structures was also similar except for the presence of inflammatory cells.

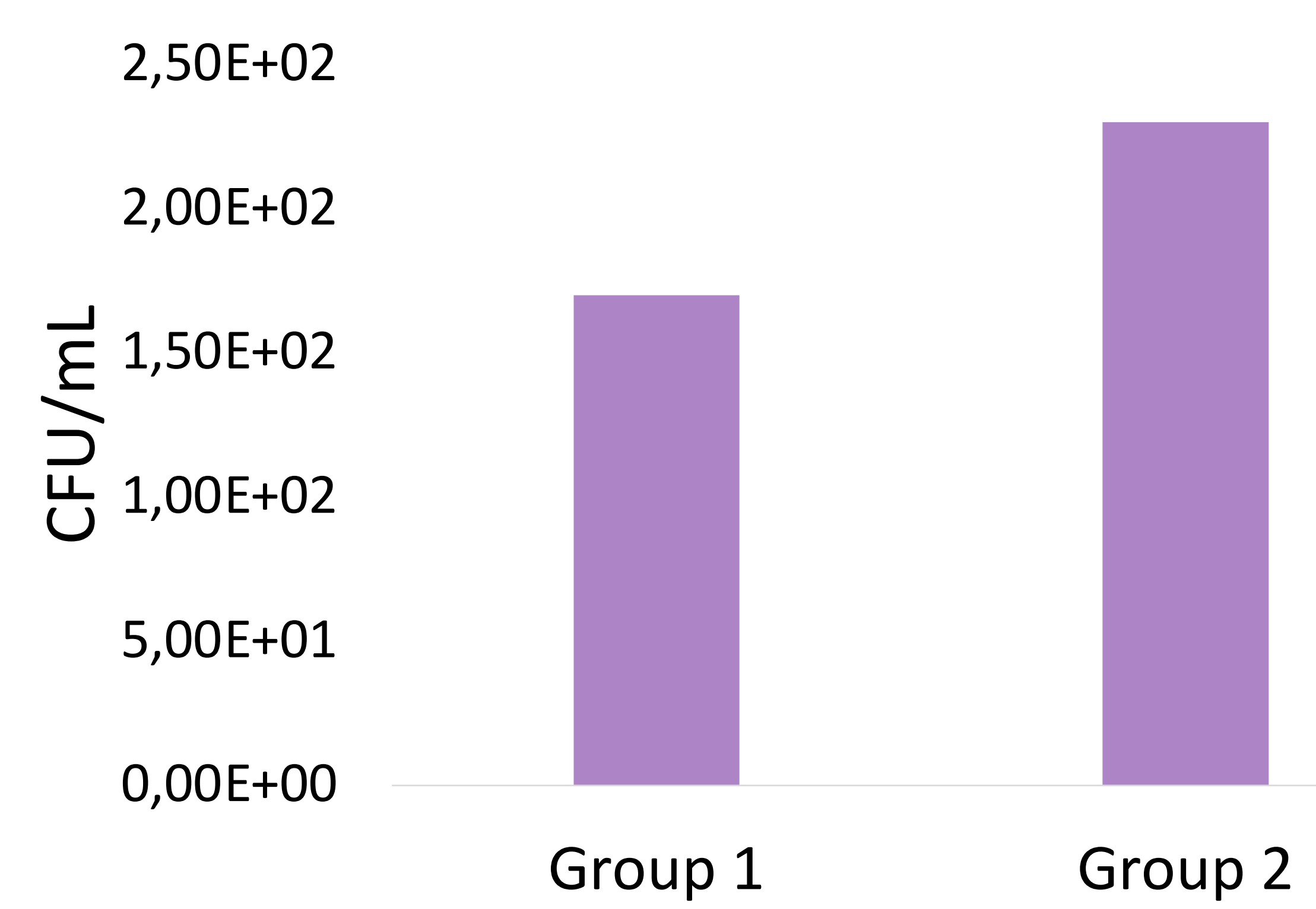

Figure 2. CFU/mL present in the skin of control group (Group 1) and treated group (Group 2).

\section{Conclusions}

Although phenolic compounds extracted from winery by-products seem to have a promising antibacterial activity in vitro, the same seems to not true in vivo.

\section{References}

Vázquez-Olivo, G.; Cabanillas-Bojórquez, L.A.; Elizalde-Romero, C.A.; Heredia, J.B. Phenolics from Agro-industrial By-Products BT - Plant Phenolics in Sustainable Agriculture: Volume 1. In; Lone, R., Shuab, R., Kamili, A.N., Eds.; Springer Singapore: Singapore, 2020; pp. 331-346 ISBN 978-981-15-4890-1.

Dev, S.K.; Choudhury, P.K.; Srivastava, R.; Sharma, M. Antimicrobial, anti-inflammatory and wound healing activity of polyherbal formulation. Biomed. Pharmacother. 2019, 111, $555-567$

This work was supported by the Associate Laboratory for Green Chemistry - LAQV which is financed by national funds from FCT/MCTES (UIDB/50006/2020). Vanessa Silva is grateful to FCT (Fundação para a Ciência e a Tecnologia) for financial support through PhD grant SFRH/BD/137947/2018. 2018 